\title{
Gelation Process of Toluene-Based bis-Urea in Cyclohexane Studied with Magnetic Resonance Imaging
}

\author{
J. Tritt-GoC ${ }^{a, *}, J$. BoguszyńskA ${ }^{a}$, M. SzWAJ ${ }^{a}$, \\ L. BOUTELliER ${ }^{b}$ AND J. JADŻYN ${ }^{a}$ \\ ${ }^{a}$ Institute of Molecular Physics, Polish Academy of Sciences \\ M. Smoluchowskiego 17, 60-179 Poznań, Poland \\ ${ }^{b}$ Laboratoire de Chimie des Polyméres, UMR 7610, Université Pierre et \\ Marie Curie, 4 place Jussieu, 75252 Paris CEDEX 05, France

\begin{abstract}
Magnetic resonance imaging was used for studies of the gelation and swelling processes of toluene-based bis-urea prepared in a form of cylindrical tablet and immersed in cyclohexane. The processes were investigated with the use of Bruker AVANCE (300 MHz) spectrometer equipped with a micro imaging probe head. The images were taken for cyclohexane protons within the bis-urea gel formed around the sample dry core at different intervals of the immersion. The mobility of the solvent molecules was estimated from the spatially resolved distribution of the spin-spin relaxation time $T_{2}$ and the spin densities $\rho$, calculated on the basis of the images obtained. It was shown that the time-evolution of the thickness of the gel layer can be well described by the power equation with an exponent equal to $0.47( \pm 0.04)$, indicating the Fickian nature of the diffusion of cyclohexane molecules.
\end{abstract}

PACS numbers: 82.70.Gg, 76.60.Pc, 82.56.Lz, 82.56.Na

\section{Introduction}

The toluene-based bis-urea belongs to a new class of low molecular weight gelators [1-8]. Such small organic molecules interact in solution (mostly in organic solvent, but also in water) with the other molecules in such a way that large supramolecular aggregates in a form of elongated fibers are created. Entanglement

*corresponding author; e-mail: jtg@ifmpan.poznan.pl 
of the fibers produces a three-dimensional network capable of trapping the solvent molecules and yielding a gel. In contrast to polymer gels, the interaction between gelator molecules is here non-covalent in nature and can take the form of van der Waals interactions, $\pi-\pi^{*}$ stacking, dipole-dipole or electrostatic interactions as well as hydrogen bonding. That new family of low molecular weight gelators can be used in practice in solar and electrochemical cells, in production of microporous aerogels or in various kinds of paints, for example.

The compound studied, toluene-based bis-urea, is an organogelator where the bifurcated hydrogen bonds between urea groups are responsible for the molecular self-assembling [9-11]. Usually the procedure for a gel formation consists of heating of the mixture of low-molecular weight gelator and a solvent in closed tube to promote homogeneity of the system, and next cooling up to trap solvent molecules in the assembled entangled network. The resulting material is considered to be organogel if upon reversion of the test tube it retains the original shape and it exhibits the resistance to flow [1]. Such process of gelation is completely thermoreversible.

In the past, most low-molecular weight gelators were found accidentally, but recently a lot of work is being performed to design novel gelators based on the urea or other molecular units $[12,13]$.

In this paper the results are reported on the use of magnetic resonance imaging (MRI) to studies of gelation phenomenon of toluene-based bis-urea immersed in cyclohexane. The MRI is the only method suitable for investigations of the dynamics of the gelation process in a real time [14-16]. The method provides a visualization of the cyclohexane molecules diffusion into the urea sample with a resolution of the order of $\mu \mathrm{m}$, so the experiment allows a quantitative description of the process.

\section{Experimental}

\subsection{Sample preparation}

The toluene-based bis-urea consists of two identically substituted urea groups connected by a toluene linker - see Fig. 1 . The synthesis of the compound was

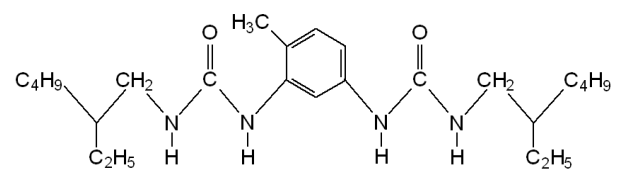

Fig. 1. Toluene-based bis-urea compound studied.

describes previously [5] and was used in the form of white flake-like powder. $0.5 \mathrm{~g}$ of such powder was compressed under hydraulic pressure of $90 \mathrm{MPa}$ to obtain the sample in the form of a cylindrical tablet with a diameter and length equal to $5 \mathrm{~mm}$ 
and $8 \mathrm{~mm}$, respectively. The tablet was glued to the bottom of a standard NMR tube (20 $\mathrm{mm}$ in diameters) to prevent movement of the sample when applying the magnetic field gradients. The gelation process started when the urea tablet was immersed in cyclohexane. The temperature of the solvent and urea tablet was maintained constant $\left(25^{\circ} \mathrm{C}\right)$. At different gelation time the cyclohexane was removed from the tube and the NMR images of the sample were taken. Such a procedure was repeated until gelation of almost the whole sample occurred.

\subsection{MRI measurements}

The measurements of the gelation process of the bis-urea in cyclohexane or equivalently the study of cyclohexane molecules diffusion into the sample under investigation were performed with a Bruker AVANCE $300 \mathrm{MHz}$ spectrometer equipped with a micro imaging probe head, the magnetic field gradient systems, and the Para Vision program. Therefore, it was possible to run the imaging experiments with the spectrometer. A single-slice multi spin-echo imaging pulse sequence was used to obtain $T_{2}$ and $\rho$ weighted images. The images were taken for a slice of $1 \mathrm{~mm}$ thick from the middle of the sample, perpendicular to the sample cylinder axis (transverse slice). The in-plane resolution of the images was $117 \mu \mathrm{m} \times 117 \mu \mathrm{m}$. The experimental condition (the value of the first echo time) was fixed in such a way that only the protons of the cyclohexane gave an NMR signal. Therefore, the ungelated part of the bis-urea did not give an NMR signal, so in the picture the black color is observed for that part of the sample.

The spatial distribution of the spin-spin relaxation time $T_{2}$ and spin densities $\rho$ were determined from the images using Carr-Purcell-Meiboom-Gill (CPMG) pulse sequence $\left(90^{\circ}-\tau-\left(180^{\circ}-2 \tau\right)_{n}\right)$ with a spacing between $180^{\circ}$ pulses of $10 \mathrm{~ms}$ and $n=32[17,16]$. The two-parameters fit of the following exponential equation:

$$
S(\tau)=S_{0} \exp \left(-\tau / T_{2}\right)
$$

to the experimental points of the echo amplitude decay was performed pixel by pixel. In Eq. (1) $S(\tau)$ is the echo amplitudes at time $\tau$ and $T_{2}$ is the spin-spin relaxation time. The parameter $S_{0}$ is equivalent to the spin densities $\rho$ (in our case to the densities of the cyclohexane protons in the particular pixel of the gel of the toluene-based bis-urea).

\section{Results and discussion}

Typical images showing the gel layer formation within the bis-urea tablet as a function of gelation time, are presented in Fig. 2. The signal intensity comes from the protons of cyclohexane confined to gel layer (white color) formed around the dry core (black color) of the sample during its exposure to the solvent. A well-defined diffusion front, moving towards the center of the tablet with increasing gelation time, can be seen. The distance between the diffusion front and the 


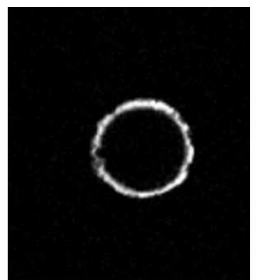

$t=15 \min$

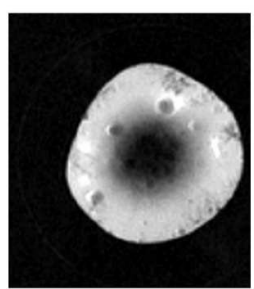

$\mathrm{t}=365 \mathrm{~min}$

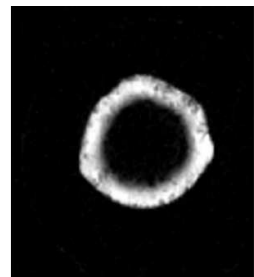

$\mathrm{t}=65 \mathrm{~min}$

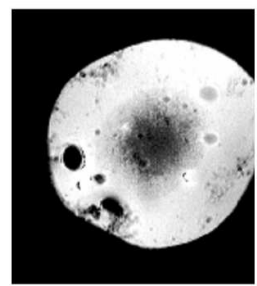

$\mathrm{t}=981 \mathrm{~min}$

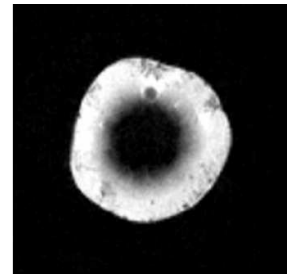

$\mathrm{t}=245 \mathrm{~min}$

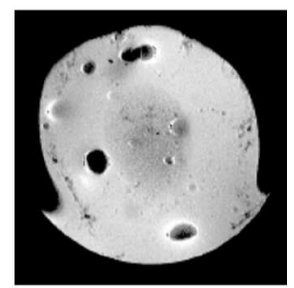

$\mathrm{t}=1261 \mathrm{~min}$

Fig. 2. Typical two-dimensional MRI images of gelated toluene-based bis-urea tablet after different times of gelation. The in-plane resolution of the images is $117 \mu \mathrm{m} \times 117 \mu \mathrm{m}$.

outer edge of the sample defines the thickness of the gel layer. The images presented in Fig. 2 show a regular growth of the gel layer, a gradual disappearance of the dry core and simultaneous increase in the tablets dimension as a function of the gelation time. Interesting features of these images are the homogeneities of the diffusion front of cyclohexane penetration within the bis-urea sample. Some bubbles seen in the images are most probably due to the air trapped in the sample during the preparation procedure.

Generally, the signal intensity of the NMR spin-echo depends on the relaxation times $T_{1}, T_{2}$ as well as on $\rho$ and $\tau[11,14,15]$. In our case the images shown in Fig. 2 are weighted by $T_{2}$ and $\rho$ only and the mathematical procedure allows one to separate an influence of these two parameters. In Fig. 3 the $T_{2^{-}}$and $\rho$-weighted images, taken after 1261 minutes of gelation of the bis-urea in cyclohexane, are shown together with the corresponding profiles. The profiles of the spin-spin relaxation times and the intensities of cyclohexane protons within the gelated tablet were taken along the white line marked across the corresponding images. The formation of the gel layer was studied up to 1261 minutes of the gelation process because our direct physical inspection of the sample shows that at this time the process seemed to be practically finished. The following conclusion resulting from Fig. 3 seems to be important. While the MRI $\rho$-weighted image shows almost identical concentration of cyclohexane across the whole sample suggesting practically final of the gelation process, the $T_{2}$-weighted image and the corresponding profile clearly shows that the gelation procedure is not finished yet. The spin-spin relaxation time of cyclohexane protons in the center of the studied sample is almost two times smaller than that in the outer parts of the sample 

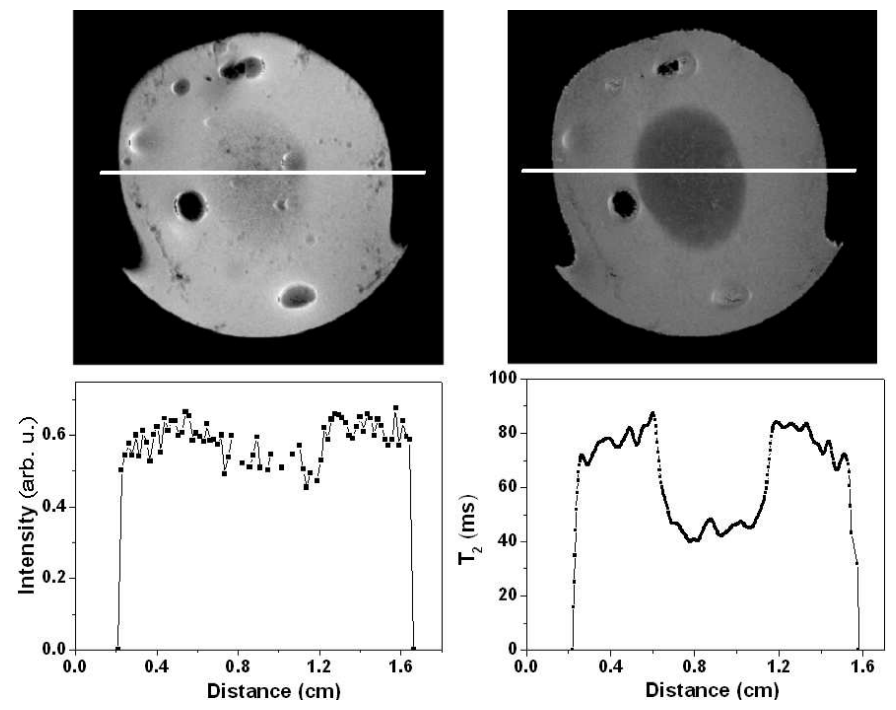

Fig. 3. Two-dimensional $T_{2^{-}}$and $\rho$-weighted images taken after 1261 minutes of gelation of the bis-urea in cyclohexane with the corresponding profiles. The profiles were taken along white line marked across the corresponding images.

(about $40 \mathrm{~ms}$ and $80 \mathrm{~ms}$, respectively). As the relaxation time directly reflects the physical environment of diffusing cyclohexane molecules, the lower values of $T_{2}$ observed within the center of the sample represent the more rigid network where the gelation procedure is still not completed.

The gelation process is accompanied by the swelling of toluene-based bisurea sample. After 1261 minutes one observes, due to the swelling process, an increase in the initial diameter of the sample of about $150 \%(5 \mathrm{~mm}$ and $12.8 \mathrm{~mm}$ for sample dry and swelled, respectively). Such a large effect was the main reason for stopping the measurements - the diameter of the NMR tube was simply too small for the swollen sample.

For each gelation time $t$, taking $c$ as the diameter of the dry cylindrical bis-urea sample and $s$ as the diameter of the swollen sample, the gel layer thickness $d$ formed around the dry core of the studied sample can be easily calculated as $d=(1 / 2)(s-c)$. The dynamics of the swelling of the matrix, represented by $d$ dependence on time $t$, can be then analyzed according to the following equation:

$$
d(t)=a+b t^{\prime \prime},
$$

where $n$ is the exponent characterizing the diffusion mechanism and $a, b$ are the constants.

Figure 4 shows that Eq. (2) quite good reproduces the experimental $d(t)$ dependence obtained for bis-urea + cyclohexane system $\left(R^{2}=0.997\right)$. From the fitting parameters the most important parameter is $n=0.47 \pm 0.04$ as its value can be directly compared to the theoretical predictions concerning the mechanism 


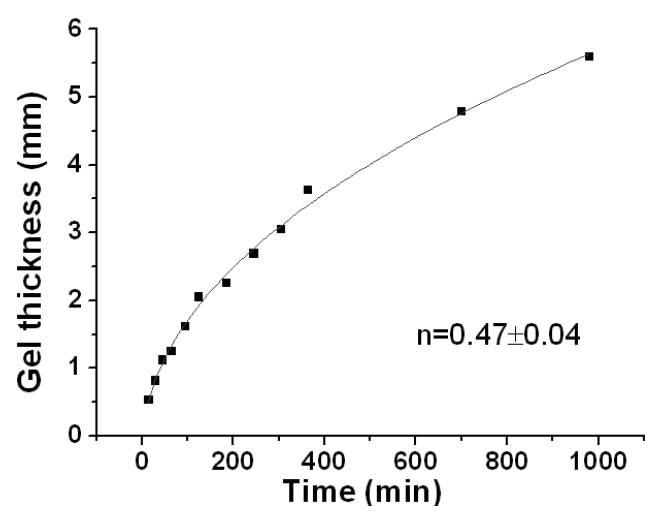

Fig. 4. Thickness of the gel layer formed on the bis-urea tablet as a function of gelation time, evaluated from MRI images. The solid line represents the best fit of Eq. (2) to the experimental points.

of the diffusion of solvent in a porous matrix. As shown in papers $[18,19]$ only two cases concern the physical reality: for $n=0.5$, where we are dealing with the Fickian diffusion and for $n=1$, where the swelling-controlled process is dominating. The value of $n$ obtained in our studies undoubtedly points out for the Fickian diffusion of the cyclohexane molecules in bis-urea matrix.

\section{Conclusion}

The presented results show that the MRI is an excellent tool for studying the gelation and swelling processes of low-molecular organic gelator immersed in nonpolar medium. The spatial spin-spin relaxation time and the spin densities were determined from the images recorded for particular gelation times. The corresponding profile of the relaxation time gives the information on the mobility of the small molecules within the gel layer. On base of that profile, as shown in our paper, one can evaluate the end of the gelation process within the whole sample. In case of studied toluene-based bis-urea + cyclohexane system, in the frame of the experimental errors, the time evolution of the gel layer thickness fulfills the $d \sim t^{0.5}$ dependence which corresponds to a simple Fickian diffusion.

\section{References}

[1] P. Terech, R.G. Weiss, Chem. Rev. 97, 3133 (1997).

[2] D.J. Abdallah, R.G. Weiss, Adv. Mater. 12, 1237 (2000).

[3] A.J. Carr, R. Melendez, S.J. Geib, A.D. Hamilton, Tetrahedron Lett. 39, 7447 (1998).

[4] S. Boileau, L. Bouteiller, F. Lauprêtre, F. Lortie, New J. Chem. 24, 845 (2000). 
[5] F. Lortie, S. Boileau, L. Bouteiller, C. Chassenieux, B. Demé, G. Ducouret, M. Jalabert, F. Lauprêtre, P. Terech, Langmuir 18, 7218 (2002).

6] O. Gronwald, E. Snip, S. Shinkai, Cur. Op. Coll. Interf. Sci. 7, 148 (2002).

[7] K. Yabuuchi, E. Marfo-Owusu, T. Kato, Org. Biomol. Chem. 1, 3464 (2003).

[8] G. Wang, A.D. Hamilton, Chem. Com. 3, 310 (2003).

[9] J. Jadżyn, M. Stockhausen, B. Żywucki, J. Phys. Chem. 91, 754 (1987).

[10] G. Wang, A.D. Hamiltob, Chem. Eur. J. 8, 1954 (2002).

[11] J.J. Kane, R.F. Liao, J.W. Laufer, F.W. Flower, J. Am. Chem. Soc. 117, 12003 (1995).

[12] Supramolecular Polymers, Ed. A. Ciferri, Marcel Dekker, New York 2000.

[13] L. Brunsveld, B.J.B. Folmer, E.W. Meijer, R.P. Sijbesma, Chem. Rev. 101, 4071 (2001).

[14] P.T. Callaghan, Principles of Nuclear Magnetic Resonance Microscopy, Oxford Sci. Publ., New York 1991.

[15] B. Blümich, NMR Imaging of Materials, Oxford Sci. Publ., New York 2000.

[16] R. Kimmich, NMR Tomography, Diffusometry, Relaxometry, Springer, Heidelberg 1997.

[17] S. Meiboom, D. Gill, Rev. Sci. Instrum. 29, 688 (1958).

[18] M. Ercken, P. Adriaensens, G. Reggers, R. Carleer, D. Vanderzande, J. Gelan, Macromolecules 29, 5671 (1996).

[19] T. Alfrey, E.F. Gurnee, W.G. Lloyd, J. Polymer Sci. C 12, 249 (1966). 